\title{
A Simple Screening Test for Cancer
}

\author{
AbulKalam M Shamsuddin* \\ Early Detection, USA
}

*Corresponding author: AbulKalam M Shamsuddin, Early Detection, Inc. 1313 Ynez Place, Coronado CA 92118, USA.

Received Date: March 16, 2021

Published Date: April 07, 2021

\begin{abstract}
Comparative and correlative studies of the pathology and pathogenesis of colon cancer in animal models and human disease have resulted in conceptualization of 'field effect" theory, and identification of a simple carbohydrate marker that is expressed early during carcinogenesis. This assimilated body of knowledge has resulted in development of a simple screening test for cancers of the lung, breast, colorectum, uterus, pancreas, and prostate. The marker galactose-N acetyl-galactosamine (Gal-GalNAc) is expressed in the cell surface and secreted glycoproteins of otherwise normal appearing tissues remote from cancer or precancerous lesions and is detected by enzymatic oxidation (10 minutes) followed by color reaction (1 minute). The high sensitivity, specificity, and cost-effectiveness of this point-of-care test makes it a great tool in our strategies for early detection, hence control of cancer. It would also reduce the number of unnecessary and expensive procedures, thereby decreasing the total national health-care cost to the societies and governments, globally.
\end{abstract}

Keywords: Screening test; Lung cancer; Breast cancer; Prostate cancer; Colon cancer; FOBT

Abbreviations: AOM: azoxymethane; FOBT: fecal occult blood test; Gal-GalNAc: galactose-N acetyl- galactosamine; GOS: galactose oxidase Schiff; MNNG: N-methyl-N'-nitro-N-nitroso-guanidine; NCI: National Cancer Institute; T-Ag or TF-Ag: Thomsen-Friedenreich antigen

\section{Introduction}

Regrettably, in 2020 approximately 10 million people were killed by cancer; now-a-days there is hardly a family that is not affected by cancer. Additionally, the healthcare cost, and loss of productivity owing to morbidity and poor quality of life, has been immensely draining to families, societies, and governments alike, everywhere. This scourge has been around for a very long time with no end in sight. As in SARS-Cov-2 now, in 1971 President Richard Nixon had declared 'War on Cancer' to combat this menace. Alas, 50 years later, conquering cancer remains an elusive goal despite progress in treatment, albeit modest. Again, akin to SARS-Cov-2, prevention is our best alternative. Cancer prevention includes (a) detection at the very early stage of the disease (secondary prevention) to reduce cancer mortality and increase the survival rate of patients; and (b) etiology prevention or primary prevention to reverse precancerous lesions or in situ carcinomas (a cancer that is still confined) to normal or stop them from progressing to invasive malignancies in populations at high risk - 'nipping in the bud'. Thus, early detection is fundamental to prevention, and the key is to find the marker which is differentially expressed in highrisk tissues (cancer and precancer) but not in normal. Biomarker(s) meeting this criterion is/are logical choice for establishing accurate methods to detect cancers at infancy; it may also help in monitoring the efficacy of chemoprevention program by serving as intermediate endpoint marker [1].

Fundamental to the success of prevention programs of any disease is the detection of the problems. It includes identification of people with existing disease and those who are at risk of developing the disease. Identification of people with cancer is relatively simple for most cancers because of signs and symptoms from the disease; the individual, now a patient seeks medical attention. Extensive 
work-up including a battery of diagnostic tests are performed and appropriate therapy is administered; regrettably, it is usually too late for many, if not for most as it does not prevent the disease. Hence the adage: an ounce of prevention is better than a pound of cure. For an effective prevention program, we must actively seek individuals with cancer or high risk thereof from an apparently healthy noncompliant population. This is done by separating i.e., screening individuals into groups with high and low probability of cancer with the help of rapid, simple, accurate, and inexpensive tests (screening tests). Implicit in the definition of screening is a promise that there is a benefit for those who participate; they will be followed with further diagnostic tests and future management of the problem. But a screening test is never intended to give the full diagnosis, hence the distinction from diagnostic tests [2]. An individual who is screening test positive will need to undergo diagnostic procedures to confirm the presence of the disease. Ideal screening tests should have a high sensitivity (proportion of diseased subjects who are test- positive) and specificity (proportion of non-diseased subjects who are testnegative), be simple and non- invasive or minimally invasive, easy to administer - therefore enjoying a high acceptability amongst populations and of course cost-effective. It would not be successful if it is shunned for discomfort, cultural, religious, or other reasons or just too expensive [2]. Currently, except for colon cancer, there are no true good screening tests for other cancers at early stages. Imaging technologies such as mammogram, chest X-ray, low dose CT etc. cannot detect early cancers, for a tumor must attain a minimum size before it can be 'visible' by these. And that is too late!

\section{The Galactose Oxidase-Schiff Test}

The Galactose Oxidase-Schiff Test is one such screening test that fits all the criteria of an ideal screening test as it is rapid, simple, non-invasive, easy to administer, and inexpensive. Its high accuracy has been consistently and reproducibly demonstrated independently by numerous investigators in three continents. That is not surprising because of extensive scientific research on carcinogenesis and operation of i) field-effect of carcinogens and ii) a marker differentially and specifically expressed during carcinogenesis, shared by both cancer and precancer, but not by normal or regenerating tissue.

\section{Field-effect}

I studied early detection of cancer using colon cancer as a model since 1975 at the University of Maryland School of Medicine as my $\mathrm{PhD}$ dissertation. The advantages of colon as a model include a) relatively easy access to samples, b) well known precancerous changes (polyps) and conditions e.g., ulcerative colitis, Crohn's disease etc.; c) well developed animal models with d) well characterized steps of carcinogenesis viz. initiation $\rightarrow$ promotion $\rightarrow$ progression; etc. Both in vivo and in vitro carcinogenesis experiments with rats and mice were performed to see how colon cancer forms in them, and to identify the earliest recognizable changes by microscopy and histochemistry $[3,4]$. Colon tissue from rats and human were cultured in the Petri dishes and exposed to the carcinogens N-methyl-N'-nitro-N-nitroso- guanidine (MNNG) for rat colon [3], and fecapentaene-12 a suspected human carcinogen, for human colon explants [5]. The findings were correlated with in vivo animal models, and ultimately to the human disease [6]: what does the human colon near and far from the cancer look like (Figure 1)?

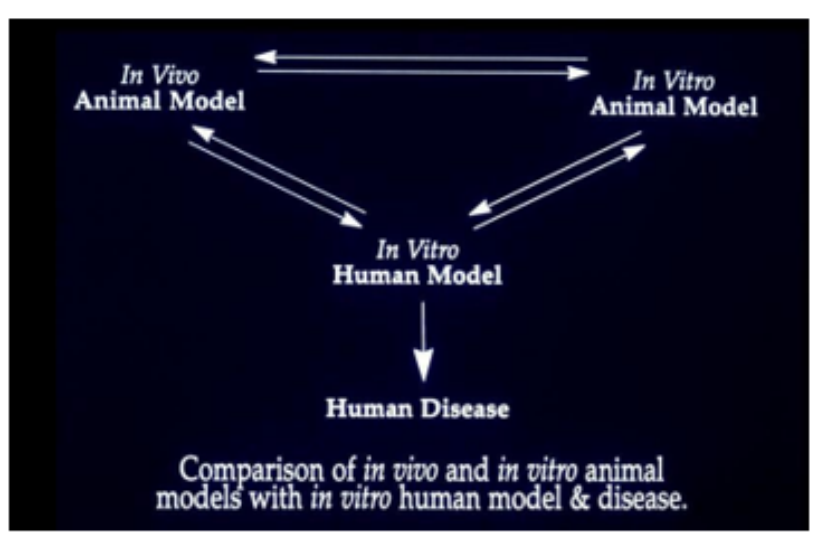

Figure 1: Schematic diagram of the approach in studying carcinogenesis and identification of the earliest changes detectable.

It has been observed that the earliest recognizable change in the carcinogen-exposed tissue is an alteration in the composition of secreted mucus that persists through precancerous and cancerous tissues. The microscopic and histochemical changes in the human colon away from cancer are identical to those seen in the colons of rats (mouse colon is distinctly different from human or rat colon [7]) treated with the carcinogen azoxymethane in vivo, or MNNG for rat explants and fecapentaene-12 for human explants in vitro in Petri dishes. These changes are therefore the earliest evidence (or markers) of cancer formation - even before a cancer has 
formed. Using high iron diamine alcian blue technique, the mucus in the colon away from the cancer that looked normal by naked eye appears as blue (Figure 2, lower frame) as opposed to black in truly normal human colon without cancer, in the upper frame (Figure 2). Also note that the colonic glands or crypts in a cancer bearing colon appear distorted - changes identical to the rat carcinogenesis models. It was reasoned that because of the generalized effect of the carcinogen throughout the entire field of the large intestine, it is most likely that the tissue away from an obvious cancer would be abnormal - Field-Effect. The carcinogens in our environment such as the air we breathe, the food we eat etc. expose the entire lung or large bowel. Therefore, it is logical that their effect would be observed throughout the exposed field. While the vast majority of the cells will undergo DNA repair, and other host defense mechanisms such as NK cell will prevent them to progress to cancer, a few cells will be promoted and even fewer progressed to precancer and cancer who may share the same early changes as the rest of the exposed tissue in the field. (Figures 2,3).

Figure 2: Normal human colon expressing sulfomucin (black) in well-formed crypt (upper panel) as opposed to distorted crypt containing blue sialomucin in normal looking mucosa away from cancer (bottom).
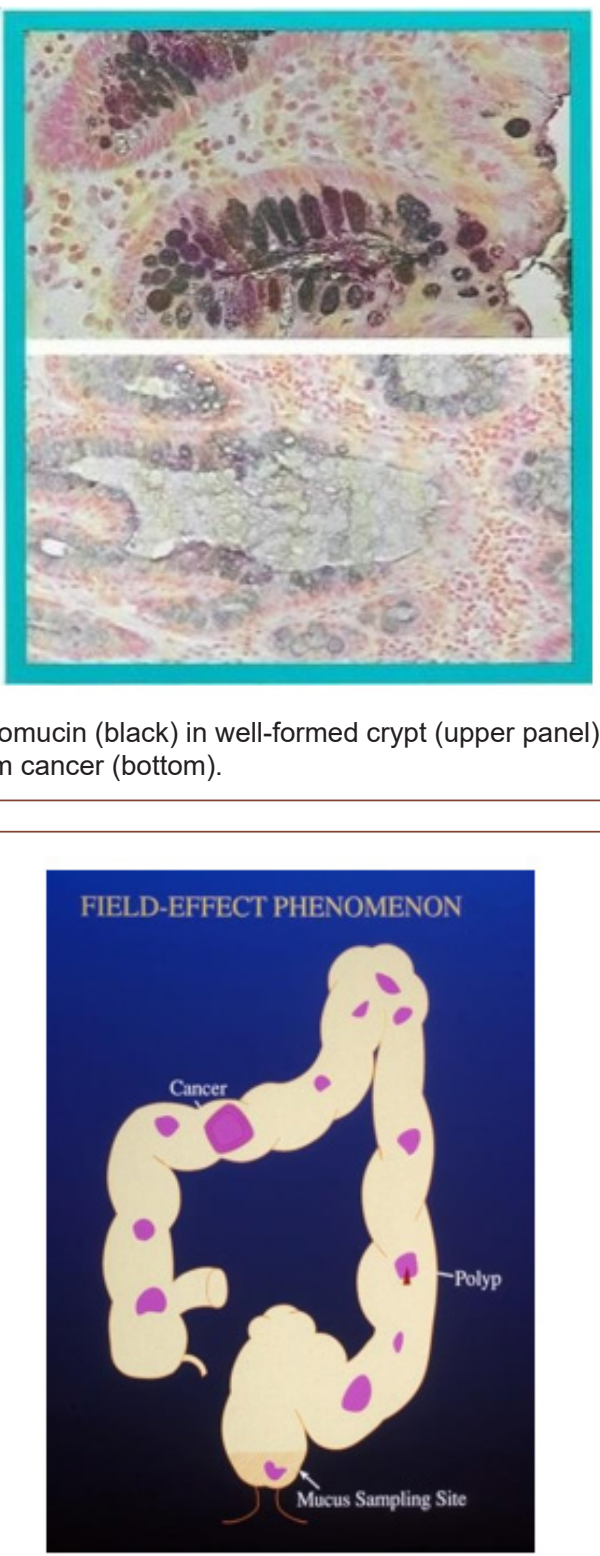

Figure 3: Schematic diagram of the Field-Effect phenomenon depicting the changes (magenta) throughout the entire 'field' of the colorectum.

Field-Effect phenomenon indicates that the entire field bears the brunt of the carcinogenic assault and expresses variable changes throughout. In the schematic drawing of the large intestine (Figure 3), it is depicted that irrespective of where a cancer or a precancerous polyp may be, areas of the otherwise normal appearing mucosa will show expression of the marker perhaps in a patchy manner (it is not practical to sample the entire large intestine to see if every millimeter has the change; hence this assumption of "patchy"). Rectum being a part of the large intestinal "field" and a convenient sampling site is therefore likely to show the 
same changes. Since digital rectal examination is a part of routine physical examination (though not practiced diligently by many primary care physicians now-a-days), it is simple and noninvasive. It also allows the physician to examine the prostate in males, and uterus, cervix, and adnexa in females. Samples from lungs such as coughed up sputum, nipple aspirate from breast, endocervical mucus, or prostatic secretion are likewise simple and noninvasive.

\section{The marker: gal-galnac}

The observed mucin histochemical change has further been identified as due to a biochemical alteration in the cell surface and secreted glycoprotein - presence of the carbohydrate moiety D- galactose- $ß$-(1-3)-N-acetyl-D-galactosamine (Gal-GalNAc, also called Thomsen-Friedenreich antigen or T- Ag, or TF antigen, though it may be different). In normal cells, a terminal sialic acid blocks the T-Ag from being recognized by the lectin peanut agglutinin (PNA), or the enzyme galactose oxidase; in cancer and precancer owing to loss of the sialic acid it is now recognizable. D-galactose oxidase specifically oxidizes C-6 hydroxyl groups of D-galactopyranose and $\mathrm{N}$-acetyl galactosamine residues of Gal-GalNAc, generating two vicinal aldehyde groups that react with basic fuchsin to give magenta/purple coloration. Thus, Gal- GalNAc can be visualized by a simple enzymatic reaction with galactose oxidase followed by Schiff's reagent, resulting in the development of a simple test for early detection, initially of colorectal cancer [8] by using rectal mucus sample.

\section{What is an Ideal Cancer Marker According to the National Cancer Institute (NCI)?}

Kelloff et al of the Division of Cancer Control and Prevention (DCCP) at the National Cancer Institute [1] outlined six criteria for intermediate endpoint biomarkers of use in chemoprevention, and here is how Gal-GalNAc and STEDi live up to those expectations:

\section{Is the intermediate biomarker differentially expressed in normal and high-risk tissue? YES!}

Figure 4 shows the expression of Gal-GalNAc (magenta) in a colon cancer but, not by the normal human colon (Figure 5). The normal tissues were obtained from healthy normal people without any cancer. Note the absence of magenta color in the mucus of normal colonic goblet cells. Also note that the colonic glands or crypts in truly normal humans appear uniform test tube shaped as compared to the those in cancer-bearing colon or in rats treated with the carcinogen azoxymethane (see Figure 4-6).

Figure 4: Colon cancer showing Gal-GalNAc.
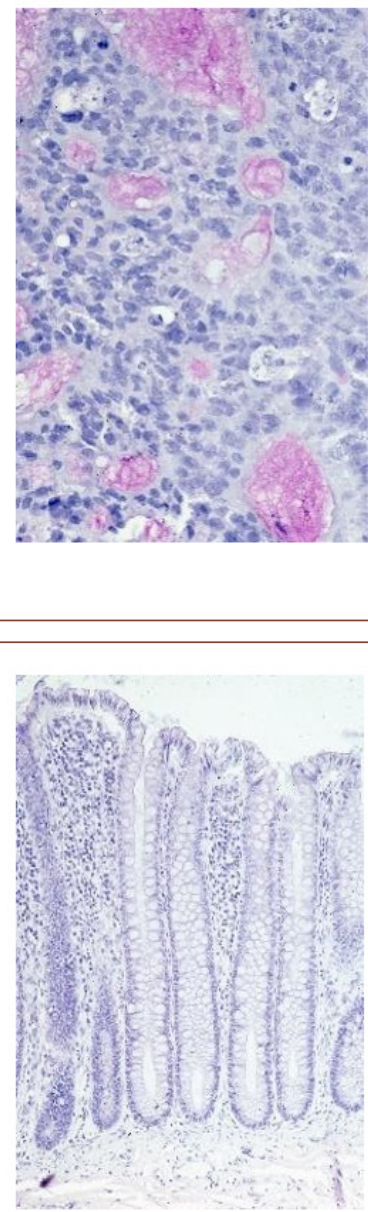

Figure 5: Normal human colon. 


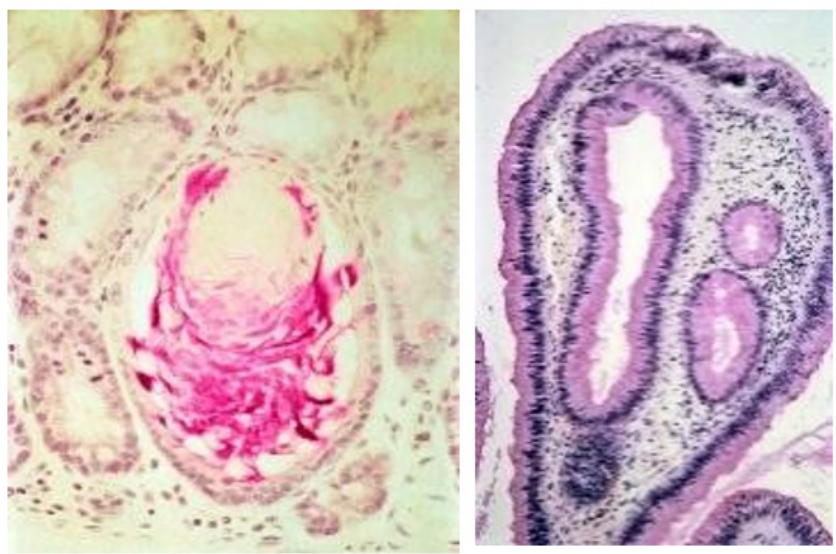

Figure 6: Rat colon expressing Gal-GalNAc (left) in a dilated crypt early in carcinogenesis 4 weeks after AOM treatment a time when a visible cancer has not developed. The right photomicrograph is of a human adenomatous polyp showing Gal-GalNAc (purple).

\section{At what stage of carcinogenesis does the marker appear?}

The earlier a reliable marker appears in the carcinogenic process, the greater is the chance for successful intervention. Answer: Gal-GalNAc is expressed very early during carcinogenesis. Not only the marker is expressed early during the carcinogenesis in rats in vivo (Figure 6 left panel) but also by human precancerous polyp (right panel appearing as purplish). The colors are slightly different owing to different batch of staining and tissue preparation. The mucus alteration is also observed in rat and human explants exposed to carcinogens in vitro [4,5]. Note the distorted appearance

Table 1: Performance Summary of GOS Test for Colorectal Cancer.

\begin{tabular}{|c|c|c|c|c|c|}
\hline Institution & Year & $\mathbf{N}$ & Specificity \% & Sensitivity \% & Reference \\
\hline U of Maryland (Pilot) Baltimore USA & 1988 & 73 & 91.5 & 100 & $\begin{array}{c}\text { Shamsuddin \& Elsayed [7] Human Pathol 19:7, } \\
1988\end{array}$ \\
\hline U of Maryland, Baltimore USA & 1988 & 240 & $\mathrm{NA}^{*}$ & 80 & $\begin{array}{c}\text { Knodell \& Schreiber Am J Gastroenterol 83:1058, } \\
\text { 1988; Gastroenterology 94: A230, } 1988\end{array}$ \\
\hline Gunma University Maebashi, Japan & 1989 & 85 & $\mathrm{NA}^{*}$ & 80 & Sakamoto et al [11] Gastroentrol Jpn 25:432, 1990 \\
\hline $\begin{array}{l}\text { Uniformed Services Uni Health Scienc- } \\
\text { es Bethesda, MD, USA }\end{array}$ & 1989 & $411^{\mathrm{a}} / 676$ & 71 & 100 & Mackett Am J Gastroenterol 84:1190, 1989 \\
\hline Red Cross Hospital Kyoto, Japan & 1990 & 94 & 81.6 & 82 & Tada et al. J Kyoto Pref Univ Med 99:681, 1990 \\
\hline Gunma University Maebashi, Japan & 1991 & $330 \mathrm{z}$ & 92.2 & $\mathrm{NA}^{*}$ & $\begin{array}{c}\text { Sakamoto Proc Am Assoc Cancer Res 32:168, 1991; } \\
\text { Cancer Biotherapy 8:49, } 1993 \text { [12] }\end{array}$ \\
\hline General Hospital Osijek Croatia & 1991 & 76 & 100 & 100 & Včev Exp Clin Gastroenterol 1:217, 1991 \\
\hline $\begin{array}{l}1^{\text {st }} \text { Medical University PLA, Guang- } \\
\text { zhou, PRC }\end{array}$ & 1991 & 77 & 90 & 89.6 & Zhou Chinese J Digestion 11:261, 1991 \\
\hline Fukuoka University Fukuoka, Japan & 1992 & 43 & $\mathrm{NA}^{*}$ & 77 & Sano Jpn J Gastroenterol Surg 25:1568,1992 \\
\hline $\begin{array}{l}\text { Fred Hutchison Cancer Res Center, } \\
\text { Seattle, USA }\end{array}$ & 1992 & $608+52$ & $\mathrm{NA}^{*}$ & 92 & Kristal Cancer Epi Biomarker Prev 1:303, 1992 \\
\hline Inst Radiation Medicine Tianjian, PRC & 1992 & 206 & 82.6 & 84.5 & Mu Proc Chinese Acad Med Sci 1993 \\
\hline $\begin{array}{c}1^{\text {st }} \text { Medical University PLA, Guangzhou } \\
\text { PRC }\end{array}$ & 1992 & 6,480 & 93.8 & 85.7 & Zhou Chinese Medical J 106:739, 1993 \\
\hline Kanazawa University Japan & 1992 & 157 & $>90$ & 69 & Watanabe Dig Endosc 4:139, 1992 \\
\hline Clinical Hospital, Split ${ }^{\mathrm{b}}$ Croatia & 2000 & 137 & 96.8 & $100^{\mathrm{b}}$ & Vucenik [13] Anticancer Res 21:1247, 2001 \\
\hline
\end{tabular}




\begin{tabular}{|c|c|c|c|c|c|}
\hline Turkey & 2002 & 100 & 88 & 90 & Koyuncu [14] Turkish J Surg. 18: 248, 2002 \\
\hline Total & & $\mathbf{9 , 4 3 4 \dagger}$ & $\mathbf{7 1 - 1 0 0}$ & $\mathbf{6 9 - 1 0 0}$ & \\
\hline
\end{tabular}

*Sensitivity or Specificity cannot be accurately determined owing to sampling bias/error. aOnly 411 of a total of 676 patients were evaluated with colonoscopy.

b96.8\% (30 of the 31) control patients had negative results i.e., no color change. The only patient of the controls that had a positive result was later found to have a sigmoid polyp, which would explain the positive test result in the initial screening; meaning that it was not really a 'false positive' reaction. 32 of $53(60 \%)$ samples collected from patients after tumor resection showed persistence of the biochemical change further supporting the Field Effect of carcinogens; 5 out of these 32 (16\%) post- operative cases with positive STEDi had tumor recurrence within a year. Thus, persistently positive test may serve as a predictor of tumor recurrence. †Additional studies involving 2,000 cases were published in Chinese in various Chinese journals by Xu, Chinese Cancer Clin J 20:661, 1993; Yang, Gui Zhou, Med Pharmacol. 17:292, 1993; Xu, Acta Acad Med Shanghai 20: 451, 1993; He, Chinese Cancer Clin J 21:434, 1994; Qu, Chinese Gastroenterol J. 4:9, 1994; Feng, Chinese J Applied Internal Med 14: 605, 1994; Lu, Acta Acad Med Shanghai 21:397, 1994. Furthermore, 91\% cancers

were detected in 54 patients tested at Roswell Park Memorial Institute, Buffalo, NY, in 1988 (unpublished).

The proof of field-Effect: Schematic diagram of the human large intestine depicting the location of the cancers detected by the rectal mucus test. That the "field-effect" phenomenon is operational is proved by the detection of cancers in the various segments of the colon remote from the rectum where the mucus was sampled from. Note that 4 of 5 cancers (80\%) of the ascending (or the right) colon, 5 of 7 (71.4\%) of the descending (or the left) colon and 29 of $33(87.9 \%)$ of the sigmoid colon were detected in the two studies by Sakamoto et al [11,12] (Figure 7). The widely used current fecal occult blood test (FOBT) for colon cancer screening has been notoriously inaccurate; "Occult blood testing is, at best an imperfect approach to the screening of colorectal cancer" concluded Dr. Ahlquist [15]. And that is not surprising since blood is not a marker of cancer or precancer. A newer test combining FOBT and DNA in stool appears to have better sensitivity and specificity albeit at a very high price.

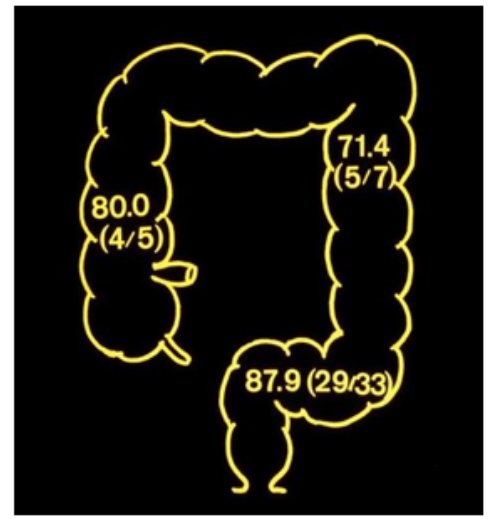

Figure 7: Colon cancer distribution.

Gal-GalNAc is a common tumor marker: The usefulness of the tumor marker Gal-GalNAc in differentiating the benign from the malignant and pre-malignant lesions of the prostate was tested [16] yielding similarly high sensitivity and specificity (vide infra). Having determined that the principle is practicable in colon and prostate, studies were conducted on other cancers. The expression of Gal-GalNAc determined in a total of 133 tissue samples from 81 cases of the carcinomas of the breast, ovary, pancreas, stomach, and endometrium and 52 cases of respective normal controls [17]. None of the 52 cases of normal tissues (except 15 cases of stomach) showed expression of Gal-GalNAc. In contrast, 100\% of adenocarcinomas from the breast (19 of 19), ovary (15 of 15), and pancreas ( 6 of 6 ), and $94.1 \%$ of stomach (16 of 17) cancers, and $91.7 \%$ (11 of 12) of uterine adenocarcinomas expressed GalGalNAc. The normal epithelia and their secretions in the vicinity of the carcinoma (within the "field") in the breast, bronchus, endometrium, and pancreatic duct also expressed Gal-GalNAc in contrast to normal tissues obtained from non-cancerous individuals, which were totally non-reactive. Thus, the tumor marker GalGalNAc recognized by galactose oxidase-Schiff sequence was highly expressed not only by a variety of adenocarcinomas but also by the apparently normal-appearing epithelia and their secretions in the vicinity of carcinomas confirming the operation of a field effect phenomenon by carcinogenic agent(s) in these organs as well, setting the stage for identification of the marker in these secretions for mass screening for these cancers too [17].

Studies on lung cancer: Twelve of 12 pulmonary adenocarcinomas expressed Gal-GalNAc. The bronchial tissue away from the cancer were available in 4 cases, all of whom also expressed the marker both in the epithelial lining cells as well as 
in the secreted mucus [17]. Coughed-up sputum therefore can be used to screen people for cancers of the lungs. Indeed, three clinical studies on lung cancer were performed on coughed up sputum [18-20]. Lai et al [18] reported the results of their study on sputum specimens from 116 healthy persons; and 216 cases of benign and malignant lung diseases were tested for the marker Gal-GalNAc. The result showed that 165 of the 182 patients $(90.7 \%$ sensitivity) with lung cancer, confirmed by cytology and histology, had positive results, whereas 22 of 116 (19.0\%) healthy controls were positive ( $81.0 \%$ specificity, Table 2 ). In 28 cases of patients whose sputum cytology showed various degrees of dysplasia-a precancerous condition that progresses to cancer, 21 were found Gal-GalNAc positive, of which 15 patients were identified to have lung cancer on further work-up! Thus, the concept of "false positive" as generally used does not apply to Gal-GalNAc and the GOS Test since they take the precancerous lesions and early cancers into consideration. In addition, three cases of early lung cancer in this study were also positive, supporting the fact that Gal-GalNAc is expressed at an earlier stage in the malignant process of the lung as well. The studies by Cox \& Miller [19] and Miller et al [20] had smaller sample size and showed sensitivities of $64.7-88 \%$ and specificities of $77.8-93.6 \%$. The test revealed 20 of 23 lung cancers among 76 patients. The other 53 patients were either healthy or had benign lung disease such as bronchitis. Even more germane to the issue of prevention is the fact that 13 of 15 cancers detected were early stage (Stage I and II). Of note is that a tumor must attain a minimum size before it can be detected by imaging techniques. And that is late! (Figures 8,9) (Table 2).

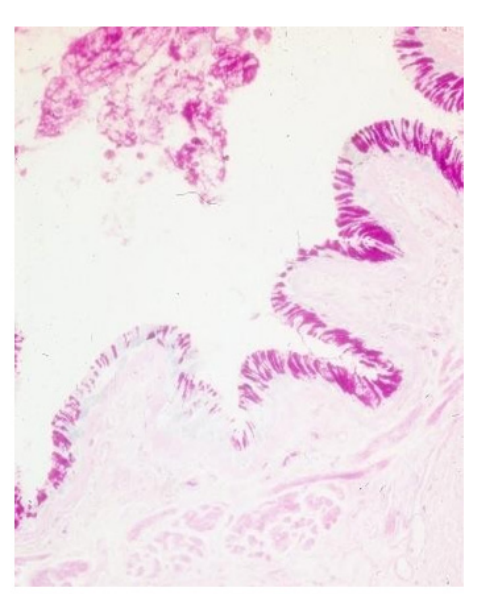

Figure 8: The photomicrograph shows histological section of such a bronchus. The magenta coloration of the mucus in the bronchial lumen as well as within the bronchial lining cells is evident.

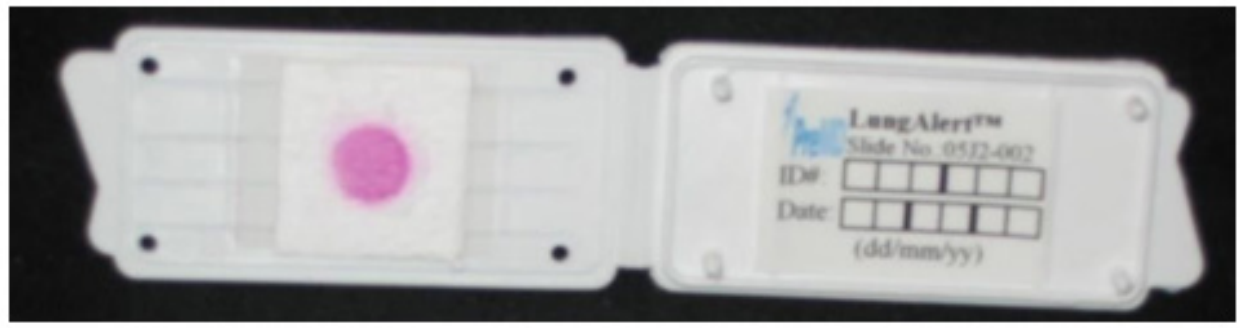

Figure 9: A sample test strip showing positive reaction (magenta color) of the sputum of a lung cancer patient. The device consists of 2 leaves made of plastic which can be folded close; on the left-hand leaf is the special white paper on which the sputum sample is placed and reacted; the opposite leaf has identification data. The size is approximately $80 \%$ of the actual device (courtesy M. Evelegh).

Table 2: Lung Cancer study.

\begin{tabular}{|c|}
\hline Lung Cancer Study \\
\hline CMU - Shenyang \\
\hline Sensitivity: $165 / 182=90.7 \%$ \\
\hline Specificity: $94 / 116=81.0 \%$ \\
\hline $21 / 28$ cases of dysplasia in sputum cytology positive $\rightarrow 15$ cancers in the follow - up study; 3 cases of early lung cancer in this study were also positive \\
\hline
\end{tabular}


Breast cancer study: Gal-GalNAc is also expressed by the normal-looking breast tissue away from an obvious cancer by way of the field-effect phenomenon [17]. The marker was positive in 19 of 19 histological specimens of breast cancer giving it a $100 \%$ sensitivity. More importantly, and germane to our screening, the ducts away from the cancers and close to the nipple also express the marker both in the cells as well as in the secretions in the ducts. Thus, the nipple aspirate from a breast harboring a cancer should express the marker. Kumar et al [21] demonstrated that Gal-GalNAc (or TF antigen) is differentially expressed in nipple aspirate, albeit using a different technique - monoclonal anti- TF antibody. Nineteen of 25 cancer patients and none of the 25 healthy controls were positive yielding a $100 \%$ specificity and $76 \%$ sensitivity. The relatively low sensitivity of the test in nipple aspirate as opposed to tissue expression may be due to the use of different techniques for identifying the marker - galactose oxidase Schiff's v monoclonal anti-TF antibody. Chagpar et al [22] also reported the utility of using nipple aspirate and Gal-GalNAc in screening for breast cancer. They investigated 23 women with biopsy confirmed, unilateral stage I or II breast cancer. They took samples (nipple aspirate by way of a suction cup attached to a syringe) from both breasts prior to surgery. Most, but not all the women were able to provide large enough fluid samples that could then be evaluated. Based on the resulting color of the test strips one could differentiate between a healthy and cancerous breast (Figures 10,11).
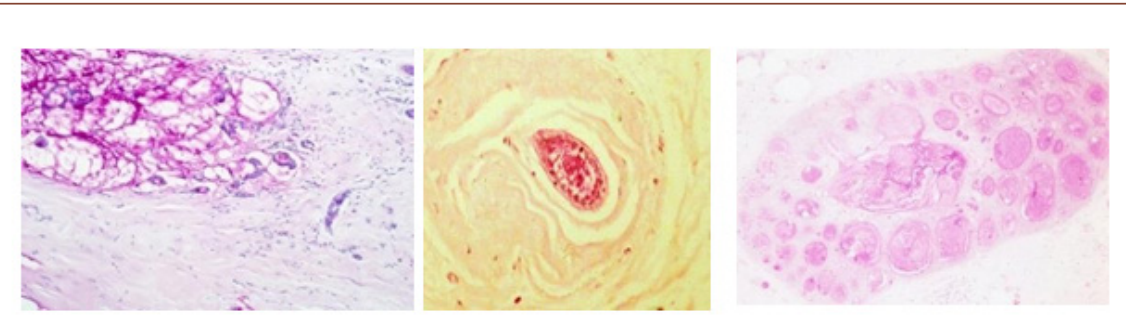

Figure 10: An invasive cancer is shown on the left and an intraductal carcinoma on right, both expressing the marker Gal-GalNAc. The center photomicrograph shows a mammary duct away from the cancer and close to the nipple expressing the marker both in the cells and in luminal secretion.

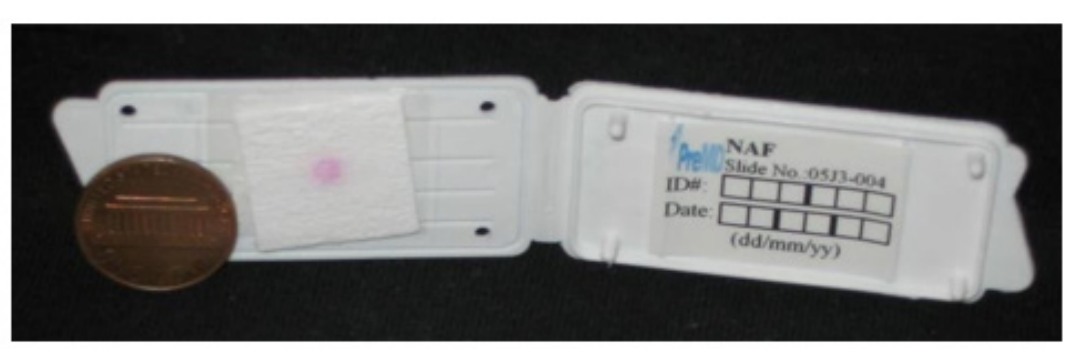

Figure 11: Positive nipple aspirate (magenta) on a sample test strip. A US penny (1 cent coin) is included for size comparison (courtesy M. Evelegh).

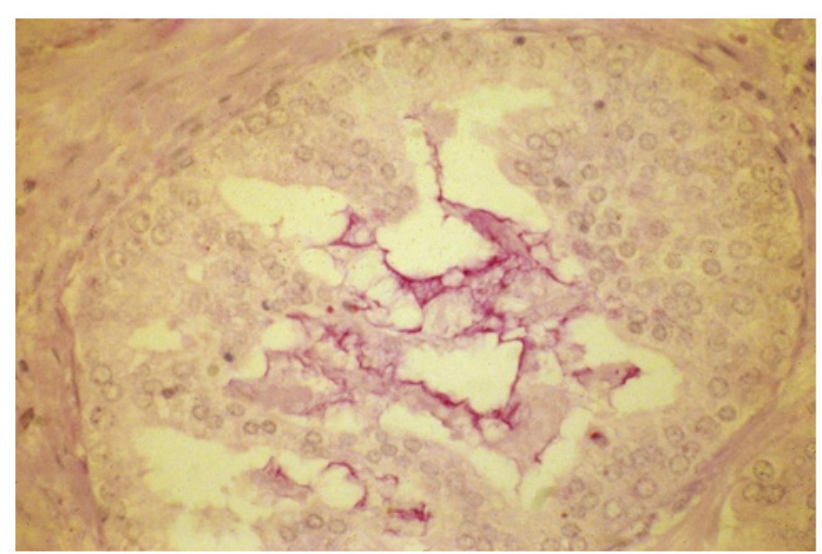

Figure 12: A focus of prostatic intraepithelial neoplasia (PIN) - considered early cancer, in a prostate with adenocarcinoma of the prostate showing Gal-GalNAc (magenta) expression in the secretions within the neoplastic glands. 
Prostate cancer study: The current screening test-PSA is inaccurate primarily because it is non-specific; its level goes up in benign conditions as well. The marker Gal-GalNAc is differentially and specifically expressed in prostate has also been observed in histological sections taken from 65 cases of adenocarcinoma [16]. While none of the 35 benign prostates and 11 foci of adenosis expressed Gal-GalNAc (100\% specificity), 62 (95.4\% sensitivity) of 65 adenocarcinomas expressed the marker. That the expression of the marker is not non-specific (meeting the NCI's criteria \#3) has been demonstrated by the absence in 25 samples of benign prostatic hyperplasia (BPH) and 11 of adenosis, neither of which are precancerous, nor were the normal prostates (10 samples) giving the marker a specificity of $100 \%$. Notably, foci of prostatic intraepithelial neoplasia when present in some of the cancer specimens were also positive (Figure 12), demonstrating the useful of this marker in identifying early cancers [16]. As for colon, lungs, and breast, testing prostatic massage secretion or seminal fluid with GOS test should yield similar results (Figure 12).

Uterine cancer: Currently there are no screening tests for uterine cancers except for maybe the Pap smear wherein a uterine cancer could be detected by chance; but it is usually advanced when the cancer cells shed from endometrium are detected in Pap smear. Expression of Gal-GalNAc with high sensitivity (91.7\%) was observed in 12 cases of uterine endometrial adenocarcinoma [17]. In all three cases where endocervical tissues was available, the endocervical mucus was positive for Gal- GalNAc. The photomicrograph (Figure 13) shows histological section of endocervix of a patient with endometrial adenocarcinoma; magenta coloration of the mucus in the endocervical glands and in endocervix is evident. Again, this supports the Field-Effect of carcinogenesis. More importantly, this makes it a simple, convenient, and non-invasive method of screening for endometrial adenocarcinoma while a gynecologist routinely collects samples for Pap smear (Figure13).

Figure 13: Endocervix.
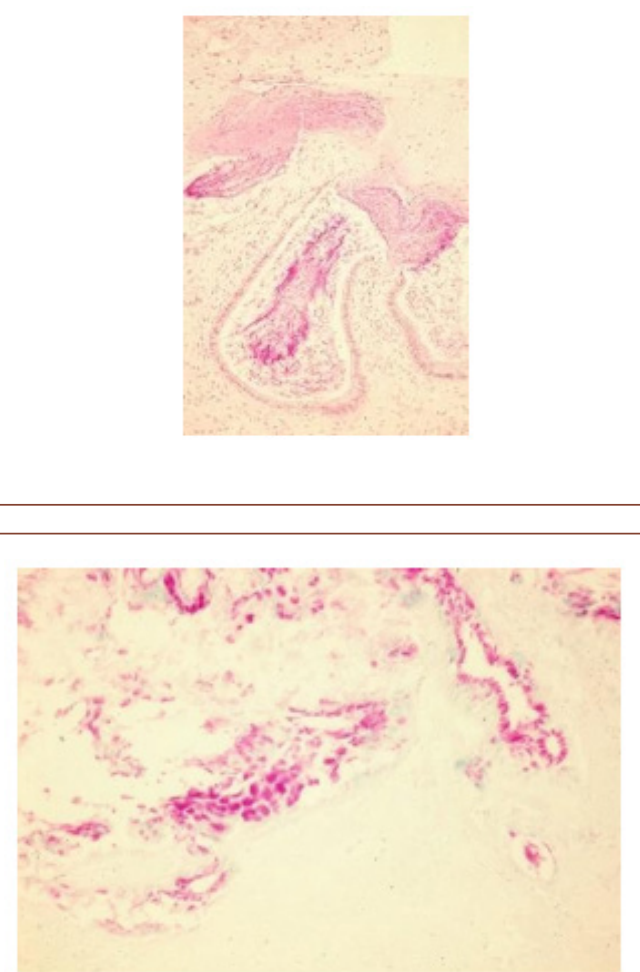

Figure 14: Pancreatic adenocarcinoma

Pancreatic cancer: Pancreatic cancer is one of the deadliest of all cancers, primarily because of its silent inception; and owing to its location, signs and symptoms do not show till very late. All 6 of 6 cases of adenocarcinoma of pancreas were positive for GalGalNAc, and the normal ducts away from the cancers also showed positive reaction [17]. Thus, GOS test could be performed on fluid samples including pancreatic juice obtained during ERCP (endoscopic retrograde cholangiopancreatography) thereby providing additional value to the screening of the cancer. Figure 14 shows an adenocarcinoma of the pancreas with magenta color in the cancerous ducts and glands. (Figure14).

\section{How easily can the marker be measured?}

Answer: GOS test for detection of the marker is non-invasive done on mucus sample obtained during routine digital rectal examination, coughed-up sputum, nipple aspirate, prostatic massage secretion, endocervical mucus etc., and the entire assay period is $\sim 15 \mathrm{~min}$. This is the only point-of-care test for breast, 
lung, colon, prostate, and uterus; the results are available before the individual is ready to leave the doctor's office. The test sample is placed on a special paper and reacted with galactose oxidase for 10 minutes, rinsed with distilled water, reacted with Schiff's basic fuchsin for 1 minute, washed with tap water and dried. A pink- magenta-purple color is positive, no color is negative (Figure 15). Positive indicates the presence of the marker Gal-GalNAc which is correlated with the presence of precancer, precancer or a high-risk thereof (Figure15).

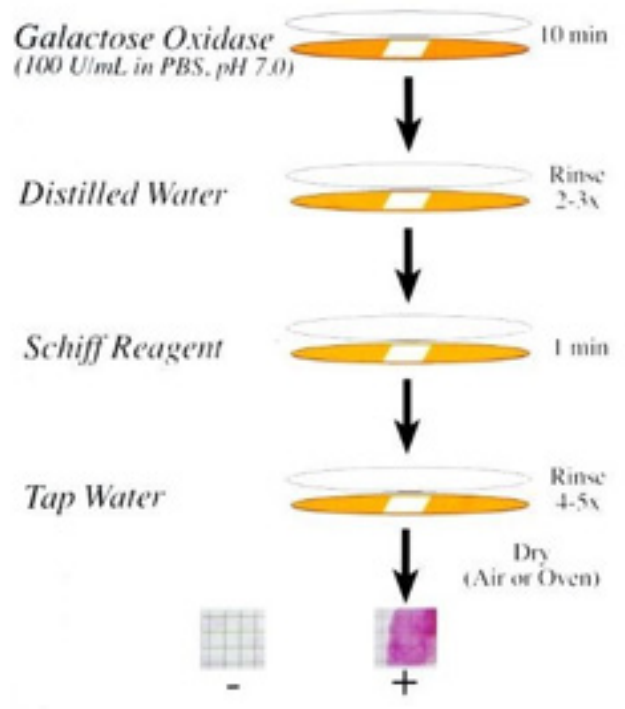

Figure 15: Steps of GOS Test.

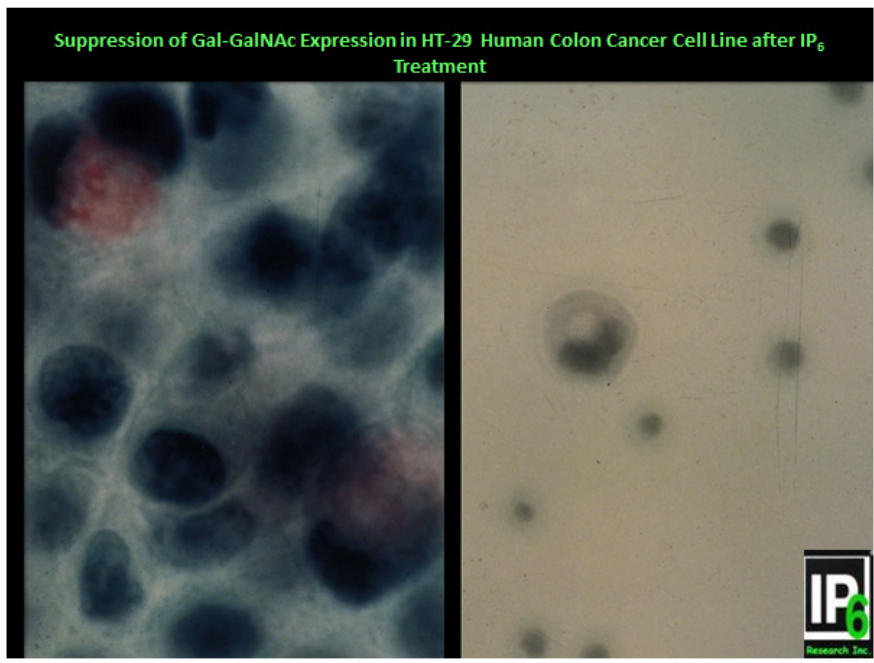

Figure 16: Control HT-29 cells (left), $\mathrm{IP}_{6}$ treated right frame.

Note: Though desirable, quantitative evaluation of the color reaction is neither practical nor meaningful as studies have shown no correlation between the intensity of color and the underlying disease.

\section{Can the marker be modulated by chemo preventive agents?}

Answer: YES! Sakamoto et al. [23] and Yang \& Shamsuddin [24] have demonstrated that; indeed Gal-GalNAc expression can be suppressed by the chemo preventive agent IP6 [25,26]. Figure 16 shows that HT-29 human colon cancer cells express the marker GalGalNAc (magenta color in mucus of cells in left panel). Following IP6 treatment, HT-29 cells terminally differentiate and produce mucin, yet not Gal-GalNAc, akin to normal goblet cells (right panel showing a differentiated HT-29 cell that has a mucus vacuole yet not expressing the marker $[23,24]$. Clinical studies are needed to validate this, but that would take a very long time. In this regard of particular importance is the study on colon cancer by Vucenik et al 
[13] where 32 of $53(60 \%)$ samples collected from patients after tumor resection showed persistence of the biochemical change; 5 out of these 32 (16\%) post-operative cases with positive GOS Test had tumor recurrence within a year. Thus, persistently positive test may serve as a predictor of tumor recurrence (Figure16).

\section{Does modulation of the intermediate biomarker correlate with a decrease in cancer rate?}

This would require a long time-years if not decades, and additional resources.

In conclusion, GOS test is a point-of-care screening test that is very simple, rapid, non-invasive, and inexpensive yet accurate for identifying asymptomatic people who may be at high risk of cancer or precancer of the lung, breast, colon, pancreas, prostate, and uterus. It is based on robust scientific background; researched and validated over three decades in $>20$ independent clinical studies in North America, Europe, and Asia. It exploits a disaccharide marker expressed early during carcinogenesis that persists in precancer and cancer and, satisfies all the practical rigid criteria set forth by NCI for an ideal marker. Given the fact that cancers of the lungs, breast, colon, prostate, and uterus comprise the most cancers, it is in the interest of public health that they are identified at a very early stage, or even before they are formed. The sooner it is used, the more lives could be saved from these cancers.

\section{Acknowledgement}

None.

\section{Conflict of Interest}

Dr Shamsuddin is a co-founder and Vice President of Early Detection, Inc., the company that will be launching the cancer screening tests in 2021.

\section{References}

1. Kelloff GJ, Malone WF, Boone CW, Steele VG, Doody LA (1992) Intermediate biomarkers of precancer and their application in chemoprevention. J Cell Biochem 16G: 15-21.

2. Shamsuddin AM (1991) Diagnostic Assays for Colon Cancer. ISBN 0-8493-6540-6, CRC Press, Boca Raton, Florida.

3. Shamsuddin AKM, Trump BF (1981) Colon epithelium II In vivo studies of colon carcinogenesis. Light microscopic, histochemical and ultrastructural studies of histogenesis of azoxymethane-induced colon carcinomas in Fischer 344 rats. JNCI 66(2): 389-401.

4. Shamsuddin AKM, Trump BF (1981) Colon epithelium III In vitro studies of colon carcinogenesis in Fischer 344 rats. N-methyl-N'-nitro$\mathrm{N}$-nitrosoguanidine induced changes in colon epithelium in explant culture. JNCI 66(2): 403-411.

5. Shamsuddin AM, Ullah A, Baten A, Hale E (1991) Stability of fecapentaene-12 and its carcinogenicity in F-344 rats. Carcinogenesis 12(4): 601-607.

6 Shamsuddin AKM, Weiss L, Phelps PC, Trump BF (1981) Colon epithelium IV Human colon carcinogenesis. Changes in human colon mucosa adjacent to and remote from carcinomas of the colon. JNCI 66: 413-419.
7 Shamsuddin AKM, Elsayed AM (1986) Ultrastructural features of normal mouse colon epithelium Unique characteristics of a species. J Submicrosc Cytology 18(4): 761-771.

8 Shamsuddin AM, Elsayed AM (1988) A test for detection of colorectal cancer. Hum Pathol 19(1): 7-10.

9 Xu H, Sakamoto K, Shamsuddin AM (1992) Detection of the tumor marker D-galactose $ß$ - [1 $\rightarrow 3]-\mathrm{N}$-Acetyl-D-galactosamine in colonic cancer and precancer. Arch Pathol Lab Med 116(11): 1234-1238.

10 Yang GY, Shamsuddin AM (1996) Gal-GalNAc a biomarker of colon carcinogenesis. Histology and Histopathology 11(3): 801-806.

11 Sakamoto K, Nakano G, Nagamachi Y (1990) A pilot study on the usefulness of a new test for mass screening of colorectal cancer in Japan. Gastroenterologia Japonica 25(4): 432-436.

12 Sakamoto K, Nakano G, Nagamachi Y (1993) Evaluation of a new test for colorectal neoplasms A prospective study of asymptomatic population. Cancer Biotherapy 8(1): 49-55.

13 Vucenik I, Gotovac J, Druzijanic N, Shamsuddin AM (2001) Usefulness of galactose oxidase-Schiff test in rectal mucus for screening of colorectal malignancy. Anticancer Res 21(2B): 1247-1255.

14 Koyuncu H, Soybir GR, Dolay K, Agrali Y, Adas G, et al. (2002) GOS test as a new screening test for colorectal tumors. Turkish J Surgery 18(5): 248-260.

15 Ahlquist DA (1992) Occult blood screening Obstacles to effectiveness. Cancer 70 (5 Suppl): 1259-1265.

16 Shamsuddin AM, Beasley WM (1994) Expression of the tumor marker D-galactose- $\beta$ - [ $1 \rightarrow 3]$-N-acetyl-D-galactosamine by premalignant and malignant prostate. Arch Pathol Lab Med 118(1): 48-51.

17 Shamsuddin AM, Tyne GT, Yang GY (1995) Common expression of the tumor marker D galactose- $\beta$-[1 $\rightarrow 3]-\mathrm{N}$ acetyl-D-galactosamine by different adenocarcinomas Evidence of field- effect phenomenon. Can Res 55(1): 149-152.

18 Li X, Dai X, He A (1995) Lung cancer screening in China [T antigen test of sputum - a new simple method for screening lung cancer] in Chinese Zhonghua Jie He He Hu Xi Za Zhi. 18(5): 285- 286.

19 Cox G, Miller JD (2004) Evaluation of the association of galactose oxidase-Schiff's reactivity in sputum with lung cancer. Presented at the American Thoracic Society Annual Meeting, Orlando, Florida, USA.

20 Miller J, Cox G, Redford K, Zawydiwski R, Evelegh M (2003) Clinical evaluation of a new screening test for lung cancer based on galactose oxidase Schiff's reactivity in sputum samples. Proceedings of the American Association of Cancer Research.

21 Kumar SR, Sauter ER, Quinn TP, Deutscher SL (2005) ThomsenFriedenreich and Tn antigen in nipple fluid Carbohydrate biomarker for breast cancer detection. Clin Cancer Res 11 (19 Pt 1): 6868-6871.

22 Chagpar A, Evelegh M, Fritsche H, Krishnamurthy S, Hunt KK, et al (2004) Prospective evaluation of a novel approach for the use of a quantitative galactose oxidase-Schiff reaction in ductal fluid samples from women with breast carcinoma. Cancer 100(12): 2549-2554.

23 Sakamoto K, Venkatraman G, Shamsuddin AM (1993) Growth inhibition and differentiation of HT-29 cells in vitro by inositol hexaphosphate (Phytic acid). Carcinogenesis 14(9): 1815-1819.

24 Yang GY, Shamsuddin AM (1995) IP-induced growth inhibition and differentiation of HT-29 human colon cancer cells Involvement of intracellular inositol phosphates, Anticancer Res 15 (6B): 2479-2488.

25 Matejuk A, Shamsuddin AM (2010) IP in cancer therapy Past, present and future. Current Cancer Therapy Reviews 6(1): 1-12.

26 Shamsuddin AKM, Yang GY (2015) Inositol \& Its Phosphates Basic Science to Practical Applications. eISBN 9781681080079 ISBN: 9781681080086 Bentham e-Book, Bentham Science Publishers, Sharjah, UAE. 